\title{
Beschäftigung im Direktvertrieb: Erwerbsarbeit mit prekären Folgen
}

Die Arbeit von Frauen im Direktvertrieb birgt eine Reihe von Prekarisierungsrisiken, die bislang weder von der Geschlechterforschung noch arbeitspolitisch aufgearbeitet worden sind. Es handelt sich um eine Beschäftigungsform, die auf einer spezifischen Konstellation aus traditionellem Geschlechterarrangement („male breadwinner model“ und Ehegattensplitting) und formaler Selbstständigkeit der Frauen beruht, die es Unternehmen wie Tupperware erlauben, Prekarisierungsrisiken und -kosten zu externalisieren. In dem Maße, in dem auch Männer zunehmend von der Erosion des Normalarbeitsverhältnisses betroffen sind, werden die im Direktvertrieb strukturell schon lange bestehenden problematischen Arbeitsbedingungen brisanter.

CLAUDIA GROß, KARINA BECKER

\section{Einleitung}

Etwas „dazu verdienen oder eine selbstständige Existenz aufbauen“, „flexibel arbeiten“ mit „geringe[n] Kosten“ und „geringe[m] Risiko“, ein „(Wieder-)Einstieg ins Berufsleben“ oder „als Angehöriger der Generation 50+ eine zweite Chance ${ }^{\text {"1 }}$ erhalten - mit diesen und ähnlichen Versprechen werben Direktvertriebe (DV) für ihre Tätigkeit. Wer für Tupperware (Haushaltsprodukte), Herbalife (Vitamine) oder PepperParties (Erotikartikel) Produkte verkauft, kann ganz ungezwungen von zu Hause aus Karriere machen und dabei auch noch umfangreiche soziale Kontakte pflegen, so die Botschaft. Angesichts der inzwischen 823.000 meist weiblichen Vertriebspartnerinnen (bis 2019 wird ein Anwachsen auf mehr als 1 Mio. erwartet), ${ }^{2}$ scheinen diese Versprechen in der Branche eine gewisse Anziehungskraft zu haben. Die Ausbreitung dieser Beschäftigungsform sagt indes noch nichts über die Arbeitsbedingungen aus. Es handelt sich um ein Beschäftigungsfeld, das weder über eine Interessenvertretung verfügt, noch lassen sich derzeit arbeitspolitische Strategien ausmachen, die die Arbeitsbedingungen von Beschäftigten aus dem DV in den Blick nehmen und auf Verbesserungen derselben zielen.

Im vorliegenden Artikel zeigen wir, dass diesbezüglich ein großer Handlungsbedarf besteht. Bei Tupperware arbeiten Frauen unter prekären, zum Teil auch ausbeuterischen Verhältnissen. Dazu gehören Löhne von unter $5 €$
brutto/Stunde (vgl. Groß 2008), völlig übertriebene Einkommensversprechen des Unternehmens (Koehn 2001; Walsh 1999), Karrieren, die mehr Schein als Sein sind (Becker 2016), hohe Fluktuationsraten (Biggart 1989; Groß 2008) und fehlende soziale Absicherung. Dass trotz dieser Probleme keine wahrnehmbaren interessenpolitischen Initiativen in diesem Feld bestehen, erklären wir mit der spezifischen Beschäftigungskonstellation im Direktvertrieb: Erstens fallen die formal selbstständigen Verkäuferinnen und Verkäufer durch das klassische Institutionengefüge der Arbeitsmarktpolitik. Das heißt jedoch nicht, dass betriebliche Strukturen hier keine Rolle spielen. Beim DV handelt es sich um ein Beschäftigungsfeld mit betrieblich vororganisierter Arbeit: So werden bei Tupperware beispielsweise Arbeitsabläufe wie das Einladen der Gäste zur Heimvorführung und deren Gestaltung, das Anwerben neuer Mitglieder usw. im Einzelnen von der Zentrale vorgegeben. Auch in anderen DV sind Produktsortiment, Produktpreise, Marketingmethoden, Kommissionen sowie sämtliche andere finanziellen Anreize von den Unternehmenszentralen festgelegt und für alle Arbeitenden bindend. In den

1 http://www.direktvertrieb.de/Karrierechancen.61.0.html (letzter Zugriff: 18.08.2016).

2 www.presseportal.de/pm/68635/3141969 (letzter Zugriff: 10.07.2016). 
regelmäßig stattfindenden Schulungen werden den im Direktvertrieb Arbeitenden das nötige Wissen, etwa zur Produktfunktionalität, aber auch verkaufsförderliche Argumente gelehrt. Weder liegen diese Aspekte in der Eigenverantwortlichkeit der dort Tätigen, noch lässt sich in dieser Hinsicht von einer Weisungsfreiheit sprechen, wie sie etwa bei selbstständigen Werkvertragsnehmern gegeben sein muss. Vor dem Hintergrund unserer Forschungen lässt sich damit sagen, dass diese Beschäftigungsvariante Merkmale von Scheinselbstständigkeit aufweist. Zweitens wurde den vorwiegend weiblichen Beschäftigten mit oft geringer Ausbildung wenig arbeitspolitische Aufmerksamkeit geschenkt. Denn während die Arbeitsbedingungen eindeutig prekäre Züge tragen, ist die Tätigkeit im Direktvertrieb oftmals durch männliche Ehepartner im „Normalarbeitsverhältnis“ finanziell und sozial abgesichert und führt daher nicht unbedingt zu prekären Lebenslagen. Die Folge dieser für den Direktvertrieb spezifischen Konstellation ist, dass die strukturell prekären Arbeitsbedingungen bislang kaum offenbar sind und die Frage kollektiver Absicherung ebenso wenig gestellt wird. Die internationalen Großkonzerne des DV mit einem deutschlandweiten Umsatz von 13,7 Mrd. $€^{3}$ haben so die Möglichkeit, im völlig mitbestimmungsfreien Raum ohne jegliches Gegengewicht zu agieren. Es gibt keinerlei Zahlen zu den Erwerbsstrukturen im DV. Gleichwohl kann angenommen werden, dass das Anwachsen dieser Vertriebsform Folgen für die industriellen Beziehungen im gewerkschaftlich organisierten Einzelhandel hat - bis hin zur Unterwanderung der dort geltenden Tarifverträge und Mitbestimmung. Während im Direktvertrieb das Beschäftigungsrisiko und die Kosten sozialer Absicherung vollständig auf die formal selbstständigen Mitglieder (und deren soziales Umfeld sowie den Staat) abgewälzt werden, müssen bei angestellten Vertriebs- und Einzelhandelsbeschäftigten diese auch von den Unternehmen getragen werden.

Vor diesem Hintergrund geht es in unserem Beitrag vor allem darum, am Beispiel der Marke Tupperware für eine Beschäftigungsform zu sensibilisieren, die jenseits der mittlerweile gut erforschten betrieblichen Felder prekärer Arbeit (Leiharbeit, Werkvertrag) liegt, aber durchaus einen Beitrag zur Ausdifferenzierung des Prekarisierungsdiskurses leistet. Es handelt sich um eine bekannte, ${ }^{4}$ aber sozialwissenschaftlich kaum beachtete und schon gar nicht erforschte Beschäftigungsform von Frauen, die in einer Grauzone zwischen abhängiger und selbstständiger Arbeit liegt und die wir daher als eine Variante von „dependent self-employment" (European Union 2013), also abhängiger Selbstständigkeit, fassen. Wir werden zeigen, dass das Engagement von Frauen bei Tupperware für diese zwar emanzipatorisches Potenzial birgt, ihre Beschäftigung zugleich aber auch mit einer Reihe von bisher weitgehend unerforschten Risiken verbunden ist.

Die empirischen Grundlagen unseres Aufsatzes beruhen, falls nicht anders angegeben, auf zwei unabhängig durchgeführten Erhebungen, die die Autorinnen 2005 und
2012 - 2015 im Direktvertrieb durchgeführt haben. Die Untersuchung im Jahr 2005 stellt die Ausgangsfrage, wie das Direktvertriebsunternehmen Tupper seine formal selbstständigen Mitglieder motiviert und kontrolliert. Dafür wurden beispielsweise Motive für den Einstieg, den Ausstieg, die finanziellen und die immateriellen Anreize für den Verbleib im Unternehmen erforscht. Die Erhebung basiert auf drei Methoden: Zunächst wurde eine offene, teilnehmende Beobachtung in der Rolle als Wissenschaftlerin durchgeführt und so insgesamt zwölf Wochenschulungen, ein regionaler „Nachwuchstag“, zwei Schulungen für neue Beraterinnen und sechs Tupperpartys besucht. Darüber hinaus war es möglich, nach den Wochenschulungen an den informellen Treffen älterer Beraterinnen teilzunehmen. Zweitens wurden zwölf leitfadengestützte Interviews mit Beraterinnen, Gruppenberaterinnen und der Bezirkshändlerin geführt sowie neun weitere durch eine Mitarbeiterin erhobene Interviews ausgewertet. Drittens erhielten die Autorinnen Zugang zu zahlreichen Unternehmensmaterialien, z. B. die wöchentlichen Informationsflyer der Bezirkshandlung, die Wettbewerbsbroschüren der Unternehmenszentrale sowie die Mitgliedervereinbarung.

Die 2012 - 2015 durchgeführte Untersuchung basiert auf zwei Instrumenten: Zum einen haben die Autorinnen mit Hilfe des Ansatzes der verdeckten teilnehmenden Beobachtung an verschiedenen Verkaufsabenden (Tupperpartys) und Gruppenmeetings der Beschäftigten teilgenommen. Dieses Vorgehen ermöglichte es am ehesten, dass das soziale Geschehen durch den Beobachtungsgang möglichst unbeeinflusst (nichtreaktiv) blieb. Zum anderen wurden 21 leitfadengestützte Interviews mit Frauen verschiedenen Alters (zwischen 23 und 55) geführt, die als Verkäuferinnen, Gruppenberaterinnen oder Bezirkshändlerinnen in verschiedenen Varianten entweder nebenbei und damit stundenweise, tageweise, sporadisch oder auch hauptberuflich bei Tupperware arbeiten oder gearbeitet haben. Beide Erhebungsmethoden gaben Aufschluss über die Motive und Erwartungen, die die Beschäftigten für ihr Engagement bei Tupperware hegen. Um noch mehr Einblick in den privaten Kontext der Beschäftigten zu bekommen, konnten zudem sechs Ehemänner für ein Interview gewonnen werden.

Für den vorliegenden Aufsatz haben wir die Forschungsergebnisse unter dem spezifischen Blickwinkel der problematischen Beschäftigungsbedingungen analysiert, um sie hier als exemplarisch für den DV vorzustellen. ${ }^{5}$ Während

3 seldia.eu/index.php?option=com_content\&view= article\&id=22\&Itemid=184 (letzter Zugriff: 01.07.2016).

4 Die Markenbekanntheit beträgt laut GfK $99 \%$; $80 \%$ aller Haushalte besitzen mindestens ein Tupperprodukt, www.direktvertrieb.de/Tupperware.153.0.html (letzter Zugriff: 05.05.2016).

5 Für eine umfangreichere Beschreibung und Analyse der zwei Erhebungen siehe Becker (2016); Groß (2008). 
sich die beiden Erhebungen in ihrer Ausrichtung, ihrem Erhebungszeitraum und ihrer Herangehensweise unterscheiden, werden in beiden Erhebungen drei Spannungsfelder der Tätigkeit deutlich, die wir näher erläutern sowie in ihrer Relevanz für den gesamten DV einordnen.

Der Artikel ist folgendermaßen aufgebaut: In den nächsten Abschnitten erläutern wir zentrale Charakteristika des DVs (2) und beschreiben Tupperware als Arbeitgeber (3). Anschließend besprechen wir drei Spannungsfelder der Tätigkeit, die nicht nur für Tupperware, sondern für den gesamten DV typisch sind (4). Im 5. Abschnitt ordnen wir die Tätigkeit in den sozialwissenschaftlichen Diskurs zum Leitbild "Guter Arbeit" ein und erklären dann, warum der DV zwischen die Stühle des (arbeitsmarktrechtlichen) Institutionengefüges fällt (6). Unser Beitrag endet mit konkreten arbeitsmarktpolitischen Forderungen und Vorschlägen für die weitere Forschung (7).

\section{Was kennzeichnet den Direktvertrieb?}

Direktvertriebe wie Avon, Amway, Herbalife, Mary Kay oder Tupperware vertreiben eine Vielzahl von Produkten an ihre Kunden: Kosmetika, Haushalts- und Wellness-Produkte, Nahrungsergänzungs- und Reinigungsmittel, Schmuck, Telefonverträge, Wein und Schokolade sind nur einige Beispiele aus der Produktpalette dieser weltweit wachsenden Beschäftigungsform (DSN 2012). Im Jahre 2013 waren mehr als 96 Mio. Menschen Mitglied eines DV und bescherten den Unternehmen einen Umsatz von circa 178,5 Mrd. US-Dollar (WFDSA 2014).

Kennzeichnend für den DV ist, dass die Verkäuferinnen und Verkäufer, oft auch Berater, Consultants oder Geschäftspartner genannt, als selbstständige Gewerbetreibende Produkte an Kunden verkaufen. Dies geschieht „direkt", das bedeutet ohne zwischengeschalteten Einzelhandel (Peterson/Wotruba 1996, S. 2). Neben dem sogenannten „Haustürgeschäft“ und Einzelberatungen ist laut Bundesverband Direktvertrieb Deutschland die Partyform die meist verbreitete Verkaufsmethode. ${ }^{6}$ Mehrere Kundinnen und Kunden kommen im privaten Umfeld zusammen, erhalten eine Produktvorführung und können anschließend Produkte bestellen bzw. direkt erwerben.

Die im Direktvertrieb Arbeitenden erhalten zum einen eine Beteiligung an den Verkaufseinnahmen. Darüber hinaus besteht bei den meisten Unternehmen auch die Möglichkeit, weitere Mitglieder anzuwerben, um so eine eigene Gruppe aufzubauen (DSN 2012). Je größer und umsatzstärker die eigene Gruppe ist, desto mehr „Superprovision“ empfangen Mitglieder (Brodie et al. 2002). Neben dem Anwerben müssen die Mitglieder dann jedoch i. d. R. auch die Mitglieder ihrer Gruppe motivieren und führen und ggfs. selbst ausbilden.

Dieses Geschäftsmodell der „dependent self-employment" kennzeichnet die formale Selbstständigkeit der Ver- trieblerinnen bzw. Vetriebler, die jedoch zugleich Merkmale aufweist, die denen abhängiger Beschäftigungsverhältnisse ähneln. Typisch ist etwa, dass die Verkaufenden in der Regel ausschließlich Produkte eines Unternehmens vertreiben. Wie gezeigt werden wird, agieren die Beschäftigten weder völlig autonom und getrennt von den Unternehmen noch sind sie in einer Weise integriert wie dies bei betrieblich organisierter Arbeit der Fall ist. Zudem schließen sie nicht etwa einen Arbeitsvertrag mit einem Unternehmen ab, sondern unterschreiben eine ein- bis zweiseitige Mitgliedervereinbarung.

Während repräsentative Studien zu den Beschäftigten des DV schlicht fehlen, geben vereinzelte Quellen Einblick in dieses in Deutschland kaum erforschte Feld: Unter Berufung auf seine Mitgliedsunternehmen geht der Bundesverband Direktvertrieb Deutschland davon aus, dass rund $83 \%$ der Mitglieder ihre Tätigkeit im Nebenerwerb betreiben, wobei unklar bleibt, wie viele Arbeitsstunden dies umfasst und inwiefern die Tätigkeit zur finanziellen Absicherung beiträgt. Auch andere Merkmale der Beschäftigten (Ausbildungsniveau, Alter, Familiensituation etc.) sind unerforscht. Aus den Zahlen des Bundesverbandes geht lediglich hervor, dass bei den im Nebenerwerb Tätigen von einem Frauenanteil von knapp $98 \%$ auszugehen ist. Es handelt sich um einen „weiblichen Beschäftigungssektor “; ${ }^{\text {; }}$ der Frauenanteil bei allen Beschäftigten liegt bei knapp $79 \% .{ }^{8}$

\section{Tuppern als Form abhängiger Selbstständigkeit}

Das Unternehmen Tupperware wurde in den 1940er Jahren in den USA gegründet und ist seit 1962 auch in Westdeutschland tätig. Seit 1996 ist Tupperware ein börsennotierter Konzern, wobei die Haushaltsproduktsparte unter der Marke Tupperware ${ }^{\oplus}$ heutzutage neben Kosmetikprodukten nur noch einen Teil des Konzerns ausmacht.

Weltweit erwirtschaftete das Unternehmen 2,3 Mrd. US-Dollar im Jahr 2015, ${ }^{9}$ in Deutschland lag der Umsatz 2014 bei 208,9 Mio. $€ .^{10}$ Ungefähr 50.000 Berater-

6 www.direktvertrieb.de/News-detail.241.0.html?\&tx_ttnews\%5Btt_news\%5D=977\&cHash=f04b4d712f06e5cde0cd6c754dcfbeef (letzter Zugriff: 19.07.2016).

7 Wir verwenden im Folgenden daher auch die weibliche Form.

8 www.direktvertrieb.de/News-detail.241.0.html?\&tx ttnews\%5Btt_news\%5D=984\&cHash=3dd41e04463d 8371b4633aaa084adb33 (letzter Zugriff: 19.07. 2016).

9 http://ir.tupperwarebrands.com/ /media/Files/T/TupperWareIR/documents/annual-reports/2015/2015-10K-filing.pdf (letzter Zugriff: 19.07.2016).

10 www.bundesanzeiger.de/ebanzwww/wexsservlet (letzter Zugriff: 19.07.2016) 
innen, ${ }^{11}$ seit Neuestem PartyManager genannt, sind in Deutschland tätig. In der Unternehmenszentrale arbeiteten 145 Angestellte bzw. Arbeitnehmerinnen. ${ }^{12}$

Wie üblich im DV, kann auch bei Tupper jede und jeder einsteigen: das Unterschreiben einer Mitgliedsvereinbarung mit dem Unternehmen bzw. der Bezirkshändlerin vor Ort genügt. Diese Vereinbarung enthält keine Arbeitszeiten, Stundenlöhne oder Angaben zum Arbeitsumfang, wohl aber die Verpflichtung, den von Tupperware vorgegebenen Standards zu folgen.

So erhalten neue Beraterinnen von den Bezirkshändlerinnen eine Einarbeitung und werden zu den wöchentlichen Montagstreffen eingeladen. Dort werden neue Produkte vorgestellt, Verkaufstipps gegeben, die monatlichen Sonderangebote erläutert und erfolgreiche Mitglieder ausgezeichnet. Die Treffen finden in der Bezirkshandlung statt, wo auch die bestellten Waren abgeholt werden können. Bei ihrer Arbeit sind die Beraterinnen gehalten, sich an die von Tupperware vorgegebenen Ablaufpläne und Muster zu halten; selbst für das Einladen der Gäste gibt das Unternehmen Formulierungen vor. Sowohl bei der Vor- und Nachbereitung der Heimvorführung als auch für die „Party“ selbst müssen sich die Beraterinnen an eine standardisierte Reihenfolge halten, die Zweifel an einer Weisungsungebundenheit - eines der zentralen Merkmale selbstständiger Arbeit - der Beraterinnen aufkommen lässt. Die Arbeitsmittel, die Produkte sowie die Gastgeberinnen- und Gastgeschenke müssen die Beraterinnen vor den Heimpartys selbst kaufen; Kataloge und Bestellformulare stellt das Unternehmen zur Verfügung. Auch die Anreiz- und Wettbewerbsstruktur von Tupperware werden vollständig durch das Unternehmen gerahmt. Obwohl nicht vertraglich festgelegt, wird zudem Wert auf ein ansprechendes Äußeres gelegt, da die Beraterinnen nicht nur sich selbst, sondern in erster Linie das Unternehmen Tupperware repräsentieren sollen.

Der Einstieg bei Tupperware als Beraterin/PartyManagerin kommt meist über persönliche Beziehungen zustande und erfolgt in der Regel, nachdem die Frauen als Gastgeberinnen eine Tupperparty veranstaltet (Langreiter 2006) oder bereits als Gast eine oder mehrere Tupperpartys besucht haben. Als Gastgeberin stellen sie ihren privaten Raum und ihre Freundschaftsnetzwerke zur Verfügung; die Produkte werden von einer Beraterin präsentiert, die im Anschluss an die Vorführung neue Tupperberaterinnen rekrutiert. Auf den Tupperpartys sind Frauen jeden Alters anzutreffen (oft verwandtschaftlich, nachbarschaftlich oder freundschaftlich verbunden). Wie bei anderen DV werden auf den Partys Produkte vorgeführt und teilweise ausprobiert. Kundinnen können anschließend die Produkte bestellen und in der Folgewoche bei der Gastgeberin abholen. Die Motive der Frauen, eine Party zu veranstalten, reichen von dem Interesse an den Produkten ${ }^{13}$ bis hin zu dem Wunsch nach Abwechslung und Geselligkeit, was vor allem von Frauen mit kleinen Kindern oder auch jenen, deren Kinder bereits aus dem Haus sind, in unseren Interviews genannt wurde.
Unter den formal selbstständigen Beraterinnen gibt es eine hierarchische Arbeitsteilung, die an betriebliche „Kaminkarrieren“ erinnert: Der Einstieg erfolgt zunächst als „einfache Beraterin“. Wer fünf aktive Beraterinnen anwirbt und diese auch betreut, steigt zur Gruppenberaterin auf. Obwohl alle Beraterinnen selbstständig sind, ist vom Unternehmen festgelegt, dass Gruppen nicht gewechselt werden dürfen - auch hier greift das Unternehmen in die Ausgestaltung der Tätigkeit ein. Aus den besten Gruppenberaterinnen deutschlandweit speisen sich wiederum die Bezirkshändlerinnen, die als Franchisenehmerinnen mit dem Unternehmen verbunden sind. Die Bezirkshändlerinnen sind verantwortlich für die Wochenschulungen, die Schulung der Neueinsteigerinnen sowie der Gruppenberaterinnen. Darüber hinaus dient die Bezirkshandlung als Lager: hier wird die Ware bestellt, durch Beraterinnen abgeholt und beschädigte Ware umgetauscht.

Anders als bei „klassischer" Selbstständigkeit ist auch das Vergütungssystem formal geregelt: Auf den Umsatz einer Party bzw. einer Bestellung beim Unternehmen erhält die Beraterin in den ersten 13 Wochen $20 \%$, anschlieBend $24 \%$ Provision. ${ }^{14}$ Davon müssen alle anfallenden Kosten wie z. B. Fahrten zur Party und zum Abholen und Ausliefern der Ware, Vorführartikel, Beteiligung an Gastund Gastgeberinnengeschenken selbst bezahlt werden. Als formal Selbstständige können sie mit keiner sozialen Absicherung oder Aufwandsentschädigung bei erfolglosem Arbeitseinsatz rechnen; die Risiken der Entlohnung unterliegen der direkten Marktförmigkeit.

11 Der Anteil der männlichen Berater liegt bei ungefähr 1\% und wird hier sprachlich nicht explizit berücksichtigt. Quelle für Anzahl Beraterinnen: Groß (2008) und http://www.faz.net/aktuell/rhein-main/die-tupperpartytrotzt-dem-wachsenden-internethandel-14348401. html?printPagedArticle=true\#pagelndex_2 (letzter Zugriff: 22.07.2016).

12 https://www.bundesanzeiger.de/ebanzwww/wexsservlet (letzter Zugriff: 19.07.2016).

13 Als Gastgeberin erhalten diese ein sogenanntes Gastgebergeschenk, wobei gilt: je mehr auf der Party verkauft wird, desto wertvoller das Geschenk.

14 www.tupperware.de/partymanager/verdienst (letzter Zu griff: 21.07.2016). 


\section{Spannungsfelder abhängiger Selbstständigkeit am Beispiel Tupperware}

\subsection{Flexibilität - begrenzt und entgrenzt}

Die zentralen Botschaften von Tupperware an (neue) Beraterinnen sind die freie Zeiteinteilung und die ideale Vereinbarkeit zwischen Familie und Beruf. Auf der Website des Unternehmens ist zu lesen: „Als PartyManager sind Sie Ihr eigener Chef! Ob tagsüber, am Abend, unter der Woche oder am Wochenende - Sie entscheiden selbst, wann und wie oft Sie aktiv sind und wie Sie Ihre Zeit einteilen. Dadurch lässt sich Ihre PartyManager-Tätigkeit perfekt mit der Familie, Ihren Hobbys oder Ihrem Beruf verbinden." 15

In unseren Erhebungen wurde deutlich, dass diese Flexibilität einer der Hauptgründe für den Einstieg ist. Vor allem Mütter mit kleinen Kindern wählen diese Tätigkeit, da sie sich besser mit der Familienarbeit vereinbaren lässt als eine klassische abhängige Beschäftigung mit festen Arbeitszeiten und der Trennung von Arbeitsund Wohnstätte. Die versprochene Flexibilität stößt jedoch in vielerlei Hinsicht an Grenzen: Während in Beispielrechnungen für offizielle Veranstaltungen zwei bis drei Stunden für eine Party veranschlagt werden, ist der tatsächliche Aufwand wesentlich höher: Geschätzte sieben bis acht Stunden (größtenteils außer Haus) sind nötig, um die folgenden Aufgaben auszuführen: Zunächst müssen Gastgeberinnen gefunden werden, oft findet ein Vorbesuch statt; zur Party kommt die Anreise- und Abreisezeit hinzu, die Waren werden auf- und abgebaut, nach der Party in der Bezirkshandlung bestellt, die Woche darauf abgeholt, zu Hause pro Gast sortiert und an die Gastgeberin geliefert. Zudem sind die Beraterinnen gehalten, an den Wochenschulungen teilzunehmen, auf denen neue Produkte eingeführt werden. Mehr noch als in jeder anderen Erwerbsarbeit sind entgrenzte Arbeitszeiten an der Tagesordnung; vielfach sind es die Beraterinnen, die sich nach den Terminen von Tupperware (Abholen der Waren), der Kundinnen (Waren ausliefern) und Gastgeberinnen richten müssen. Die versprochene Flexibilität ist damit deutlich eingeschränkt und nur um den Preis entgrenzter Arbeitszeiten möglich: Wer tatsächlich zwei bis drei Partys pro Woche ausrichtet, arbeitet ungefähr im Umfang einer Halbtagsstelle. In unseren Erhebungen sind es somit gerade die kinderlosen Beraterinnen, die zu den besten in der Bezirkshandlung gehören. Eine ältere Beraterin, deren Kinder schon lange aus dem Haus sind, erzählt über ihren Arbeitsaufwand für die im Schnitt drei bis vier Partys pro Woche: „Ja. Ich darf die Stunden nicht rechnen. Ich arbeite von morgens bis abends oder bis nachts viel für Tupper." (Interview Y,\#21)

\subsection{Hervorragende Einkommensmöglichkeit mit unbedeutendem Verdienst}

Neben dem Flexibilitätsversprechen wirbt Tupper mit einem attraktiven (Neben-)Einkommen, das weitgehend selbst bestimmt werden kann: Während im Angestelltenverhältnis das Einkommen oft festgelegt ist, kann bei Tupper sowohl sehr wenig als auch viel verdient werden - je nach Performance der Beraterin.

Nach unserer Erhebung fällt es den meisten Beraterinnen schwer, das (ursprünglich) erwünschte Einkommen zu erzielen. Gründe hierfür sind neben dem tatsächlichen Zeitaufwand pro Party (s. o.) und der Schwierigkeit, Gastgeberinnen zu finden, die vom Unternehmen festgelegte Provisionsstruktur. Nach ihrem Stundenlohn gefragt, wurde in unseren Erhebungen deutlich, dass die meisten Beraterinnen diesen nicht berechnen. Eine langjährige Beraterin gibt an: „Kann ich ihnen gar nicht sagen. ... Kann man nicht! Weil wenn man überlegt: Die Bestellungen machen, das Packen. Also mein Mann sagt manches Mal schon: ,Du hast einen Stundenlohn, wenn es viel ist, vielleicht von $5 €$ oder nicht einmal'. Aber so darf man es halt nicht sehen: Tupper muss Spaß machen, sonst hört man es wieder auf.“ (Y,\#24). Obwohl wir auch mit Beraterinnen gesprochen haben, die sich zufrieden äußerten und eine Beraterin $20 €$ brutto pro Stunde angab, schätzten die meisten ihren Stundenlohn auf $5 €$ brutto pro Stunde. Diese Höhe entspricht auch unseren eigenen Berechnungen, die auf folgenden Annahmen basieren: Bei einer Party von $250 €$ Umsatz (Bundesdurchschnitt laut befragter Bezirkshändlerin), erhält eine Beraterin 24\%, also $60 €$. Davon gehen ungefähr $15 €$ Fahrtkosten und $10 €$ Eigenbeteiligung für Gast- und Gastgeberinnengeschenke ab. Übrig bleiben für einen geschätzten Aufwand von 6-8 Stunden somit $35 €$ brutto, vor Steuern und ohne Sozialversicherungsbeitrag. Eine Beraterin, die seit der Arbeitslosigkeit ihres Mannes auf den Verdienst durch den Verkauf von Tupperware angewiesen ist, bewertet das Verhältnis zwischen Aufwand und Nutzen kritisch: „Letzte Woche hatte ich eine Vorführung, die ging länger als vier Stunden. Verkauft habe ich für $170 €$, dann kamen noch eine Stunde Hin- und Rückweg dazu, die bin ich morgen auch wieder unterwegs, wenn ich die Ware ausliefere. Das ist ganz klar ein Minusgeschäft. Verdient hat daran nur Tupperware.“ $(\mathrm{X} \#, 18)$.

Die enttäuschten finanziellen Erwartungen erklären die hohe Abbruchrate von Beraterinnen: Die meisten Mitglieder steigen bei Tupper, ebenso wie bei anderen DV (Groß 2008), innerhalb eines halben Jahres wieder aus. Lediglich eine kleine Gruppe, die zu den Spitzenverkäuferinnen gehört, bezeichnet ihr Einkommen als gut bis sehr gut. Andere bleiben aus den bereits erwähnten nicht-monetären Gründen bei Tupper. Dieses Motiv kann sich jedoch dann in sein Gegenteil

15 www.tupperware.de/partymanager/vorteile/freiezeiteinteilung (letzter Zugriff: 22.07.2016). 
verkehren, wenn mit dieser Beschäftigungsform Geld verdient werden muss, weil das Einkommen des Partners weggebrochen oder unsicher geworden ist. In dem Moment, in dem sich etwa der bisherige Hauptverdiener in prekären (Arbeits-)Verhältnissen wiederfindet, ändern sich die Funktion von Tupper und damit die mit der Arbeit verbundenen Anforderungen. Für die Betroffene wird die Arbeit bei Tupper damit zu einer Zerreißprobe, die arbeitsbedingte psychische Beanspruchungen nach sich ziehen kann (Becker 2016).

Es lässt sich somit festhalten, dass die Höhe des Einkommens formal nicht durch das Unternehmen gedeckelt ist: Wer mehr Umsatz erwirtschaftet, erhält mehr Provision. In der Praxis zeigt sich jedoch, dass die Wahrscheinlichkeit, seinen Lebensunterhalt aus der Tätigkeit erwirtschaften zu können, eher gering ist. Aus der Erhebung wurde außerdem deutlich, dass die Tätigkeit von den meisten lediglich als Ergänzung zum Haupteinkommen des (Ehe-)Partners gesehen wird (Becker 2016; Groß2008). Die relative Attraktivität der Tätigkeit speist sich somit aus anderen Quellen: dem Spaß an der Abwechslung, den fehlenden beruflichen Alternativen für auf dem Arbeitsmarkt benachteiligte Gruppen und, wie im nächsten Abschnitt beschrieben wird, der spezifischen Anerkennungs- und Motivationskultur des Unternehmens.

\subsection{Zwischen Motivationskultur und Scheinemanzipation}

Tupperware richtet sich, ähnlich wie die Vielzahl anderer DV im Haushalts- und Kosmetikbereich, auf Frauen - sowohl als Kundinnen als auch als Beraterinnen. Das Modell DV gibt ihnen die Möglichkeit, aus ihrer Hausfrauen- und Mutterrolle nicht ausbrechen zu müssen, sondern diese mit dem Wunsch nach Anerkennung verknüpfen zu können. Trotz durchschnittlich geringer Einkünfte betonen die meisten Beraterinnen, dass sie den Umgang mit Kundinnen schätzen, vor allem aber, dass Erfolge belohnt und „beklatscht"werden.

Dem Bedürfnis nach Anerkennung und Wertschätzung vieler Frauen wird durch eine im DV ausgeprägte „Motivationskultur“ (Biggart 1989) entsprochen. Das zeigt sich u. a. daran, dass kein Mitglied offen kritisiert, sondern nur gelobt wird und besonders gute Beraterinnen auf der Bühne der Wochenschulung geehrt werden (vgl. Becker 2016; Groß 2008). Die Motivation der Beraterinnen soll durch die Anerkennung der Gruppe und unterstützende Preise stimuliert werden. Letztere bestehen je nach Umfang und Zeitdauer des von der Zentrale ausgelobten Wettbewerbs aus materiell eher wertlosen Produkten (Messlöffel, Plastikblumen etc.) oder auch aus größeren Preisen (Haushaltsprodukte, Kinderspielzeug, Fahrrad, Teilnahme am Jahresseminar, Kurzreisen etc.).

Besonders attraktiv erscheinen das Lob und die (im-) materiellen Auszeichnungen vor dem Hintergrund der privaten Situation der Beraterinnen: Wer außer bei Tupper „nur“ Hausfrau und Mutter ist, vermisst die Anerkennung der eigenen Arbeitsleistung. Eine Beraterin, inzwischen Gruppenberaterin, erläutert dies: „Also die Anerkennung, okay, man bekommt sie zu Hause nicht. Es sagt keiner: ,Super Mama, du kannst ja mit dem Staublappen im Mund noch staubsaugen, kannst noch spülen und telefonierst noch!' Wer sagt das? Kein Mensch sagt das! Und [... bei Tupper] kriegst du wirklich stetig ,danke' gesagt, für das was du, du selbst dir erarbeitet hast.“ (X\#,23) Während also im privaten Umfeld Hausund Familienarbeit als selbstverständlich gelten, wird bei Tupper der Arbeitseinsatz sichtbar gemacht und belohnt. Diese nicht-monetäre Belohnung scheint die materiellen Verluste (zeitweise und unter bestimmten Bedingungen) zu kompensieren.

Längerfristig kann sich die Tätigkeit jedoch als frauenspezifischer Fallstrick erweisen. Eine langjährige Beraterin berichtet: „[N]ein ... wenn ich heute anfangen würde/sollte, würde ich nicht mehr anfangen. Schon aus dem Grund: Sie sind auch nicht abgesichert. Sie sind ja nicht rentenversichert und, und, und, ja? Ich würde sogar behaupten, das ist eine Ausbeutung der Hausfrau, ja?“(Y,\#21) Ein weiterer durch die als weitgehend positiv erlebte Motivations- und Anerkennungskultur verdeckter Aspekt ist demnach, dass Karrieren bei Tupper, wie so oft im DV, für die meisten Mitglieder nicht nachhaltig sind - weder bezüglich der Tätigkeitsdauer, der Höhe der Entlohnung, der Absicherung im Alter noch der Anerkennung auf dem „ersten Arbeitsmarkt“, wenn ein Wechsel in eine abhängige Beschäftigung angestrebt wird. Die Charakteristika der Arbeit führen eher dazu, dass eine "gendered culture" perpetuiert und weibliche Arbeit weiterhin abgewertet wird (Williams/Bermiller 2011). So erhöht die fehlende Sozialversicherung die Abhängigkeit der Tupperberaterinnen von ihrem Partner. Wer während der Familien- und Tupperphase nicht von sich aus Geld zur eigenen Absicherung zurücklegt bzw. legen kann, muss damit rechnen, im Falle einer Scheidung und bei Krankheit oder Arbeitslosigkeit des Partners sozial abzusteigen. Die fehlende gesellschaftliche Anerkennung hat zur Folge, dass selbst eine jahrelang (relativ) erfolgreiche Tätigkeit den eigenen Lebenslauf nicht aufwertet, sondern von Arbeitgebern auf dem ersten Arbeitsmarkt eher als Manko bewertet wird, sodass eine Rückkehr in den Ausbildungsberuf oder der Wechsel in eine andere, besser bezahlte Beschäftigung sich bei längerem Verbleib im DV für unsere Interviewpartnerinnen stets schwieriger gestalten. Die Anerkennungskultur des Unternehmens, z. B. Lob während der Wochenschulung, ein Tupperprodukt oder ein Kinderfahrrad als extra Belohnung, mögen kurzfristig attraktiv und sogar lukrativ erscheinen; gleichzeitig zementieren sie jedoch die Abhängigkeit vom männlichen Haupternährer und stellen ein strukturelles Risiko für weibliche Altersarmut dar (Schulze Buschoff 2011).

\section{Beschäftigung im Direktvertrieb - Gute Arbeit oder prekärer Job?}

Geringfügige Bezahlung, begrenzte und entgrenzte Flexibilität sowie eine Motivationskultur, die das emanzipatori- 
sche Potenzial eher unterläuft: Wie lassen sich diese Befunde in den sozialwissenschaftlichen Diskurs zum Leitbild „Guter Arbeit" einordnen? Zur Beantwortung dieser Frage orientieren wir uns an dem von Castel (2000) vorgeschlagenen Zonenmodell zur Beschreibung der Arbeitsgesellschaft. Prekäre Arbeit wird darin zwischen der sich durch geschützte Normalarbeit auszeichnenden Zone der Integration und der Zone der Entkopplung verortet, in der sich vornehmlich die dauerhaft vollständig von Erwerbsarbeit ausgegrenzte Bevölkerung befindet. Als prekär wird ein Arbeitsverhältnis dann bezeichnet, wenn die Beschäftigten deutlich unter ein Einkommens-, Schutz- und Integrationsniveau sinken, das in der Gesellschaft als Standard definiert und mehrheitlich anerkannt wird (siehe auch Manske/Merkel 2009; Mayer-Ahuja 2003). Bilanzieren wir das Prekarisierungspotenzial der Arbeitsbedingungen bei Tupperware anhand der von Castel (2000) vorgeschlagenen sechs Dimensionen kommen wir zu folgendem Ergebnis:

(1) Hinsichtlich der materiell-reproduktiven Dimension lässt sich die Arbeit bei Tupper als eindeutig prekär definieren. Die von einigen vorgeschlagene Differenzierung, wonach prekäre Erwerbsarbeit nicht in jedem Fall prekäre Lebenslagen nach sich zieht, ${ }^{16}$ trifft zwar für viele der von uns befragten Frauen zu, ändert aber nichts an der Bewertung der Arbeitsbedingungen bei Tupper oder anderen Tätigkeiten im DV (Koehn 2001; Walsh 1999).

(2) Eindeutig prekär ist die Arbeit mit Blick auf die institutionelle Dimension: „Normale“ Standards bei der Partizipation an industriellen und sozialen Schutzrechten (soziale Sicherungen, Arbeitsrecht, Recht auf kollektive Interessenvertretung) werden nicht nur unterschritten, sondern sind gar nicht erst vorgesehen.

(3) Bei der Bestimmung der sozial-integrativen Dimension ist eine differenzierte Argumentation geboten. Der DV ermöglicht es vielen Frauen zwar überhaupt erst, soziale Netzwerke außerhalb ihrer Privatsphäre zu pflegen, gleichwohl erfordert es ihre Arbeit bei Tupper, dass private Netzwerke für berufliche Zwecke genutzt und instrumentalisiert werden. Die Etablierung befriedigender Sozialbeziehungen bei der Arbeit wird damit zum Teil ad absurdum geführt; es kann sogar von einer Zerstörung sozialer Beziehungen gesprochen werden (Lan 2002; Walsh 1999).

(4) Die Aussichten auf eine dauerhafte Existenzsicherung durch diese erwerbsbasierte Beschäftigung sind unseren Erhebungen nach ebenfalls gering: Enttäuschte Einkommensperspektiven haben zur Folge, dass viele Frauen nach einem halben Jahr diese Erwerbsform beenden. Eine derartig temporäre Beschäftigung schränkt die Möglichkeiten einer zukunftsorientierten Lebensplanung deutlich ein. Hinzu kommt, dass sich eine langjährige Beschäftigung bei Tupper als nachteilig erweisen kann: Durch die fehlende gesellschaftliche Anerkennung der Tätigkeit verschlechtern sich die Chancen auf ein Normalarbeitsverhältnis und verstärken so die Abhängigkeit vom männlichen Haupternährer und das für Solo-Selbstständige ohnehin schon hohe Risiko auf Altersarmut.

(5) Hinsichtlich der sozial-moralischen Dimension zeigen unsere Erhebungen, dass viele Frauen versuchen, mit ihrer Arbeit bei Tupper Anerkennungsdefizite zu kompensieren. Während repräsentative Erhebungen unter den Beschäftigten bei Tupper sowie im gesamten DV fehlen, lässt sich aufgrund unserer Erhebungen vermuten, dass dies - im ganz wörtlichen Sinne - zum Teil gelingt: Die in den Gruppenmeetings sowie auf den Partys gezollte Anerkennung ist eine der Hauptmotive für die Fortsetzung der Tätigkeit. Die oft fehlende oder nur gering ausfallende materielle Anerkennung dagegen stellt einen der Hauptgründe dar, dass Frauen ihre Tätigkeit beenden. In unseren Erhebungen zeigt sich zudem, dass - wenn Tupper zu einem „Brotjob“ wird, damit also Geld verdient werden muss, - mit dieser Tätigkeit auch andere gesundheitsrelevante Belastungen einhergehen. Unter eventuell neuen familiären Bedingungen (Arbeitslosigkeit des Mannes) wird es für die Frauen schwerer, sich dem Verkaufsdruck zu entziehen (ausführlich dazu Becker 2016). Darüber hinaus lässt sich festhalten, dass die gezollte Anerkennung sich weitgehend auf das Unternehmen selbst sowie die eigenen Kundinnen beschränkt. Außerhalb des Unternehmens sowie im gesellschaftlichen Diskurs wird diese Beschäftigungsform dagegen vielfach als typische „Frauenarbeit“ deklariert, die hinsichtlich der sozial-moralischen Dimension hinter die männlich geprägte Normalarbeit zurückfällt (Williams/Bermiller 2011).

(6) Ähnliches lässt sich für die sinnhaft-subjektbezogene Dimension konstatieren: Die Einordnung dieser Tätigkeit als „non-work-related activity” (ebd.) und die Betonung intrinsischer Motivstrukturen sind Strategien, mit denen der Erwerbsarbeitscharakter bei Tupper verschleiert wird (Groß 2008; Lan 2001). Im Grunde wird damit die Sinnstiftung in den Freizeitbereich verlagert und das besagte Image als „Frauenarbeit“ zementiert.

Zusammenfassend zeigt die Einordnung in Castels Zonenmodel, dass die Arbeitsbedingungen deutlich prekäre Züge aufweisen und somit für die Beschäftigten vielfältige Prekarisierungsrisiken bergen. Gleichzeitig, und dies wird aus unserer Erhebung ebenfalls deutlich, lässt sich die Gruppe der Direktvertrieblerinnen in ihrer Gesamtheit nicht als prekär bezeichnen. Der prekäre Job bei Tupper führt keineswegs zwangsweise zu prekären Lebenslagen. Dieses ambivalente Ergebnis weist unserer Meinung nach auf ein spezifisches und zugleich konstitutives Merkmal des DV hin: Im DV wird mit (verheirateten) (Haus-)Frauen und Müttern eine Zielgruppe von Beschäftigten angesprochen, die

16 Vgl. etwa die jüngste Debatte in Sozialismus 43 (4), S. $20 \mathrm{ff}$. 
angesichts des noch stets vorherrschenden „male breadwinner model“ ihre Absicherung oft außerhalb der Tätigkeit findet. Ohne auf repräsentative Erhebungen zurückgreifen zu können, vermuten wir also, dass die soziale Sicherung hier nicht durch die Beschäftigung selbst, sondern zum großen Teil durch Ehemänner, weitere (abhängige) Beschäftigung und durch das in Deutschland bestehende Ehegattensplitting sowie im Falle von Altersarmut staatlich gewährleistet wird. Für die Unternehmen bedeutet dies, dass sie den gesamten Vertrieb ihrer Produkte kostengünstig mit formal Selbstständigen betreiben lassen können, da für diese weder Sozialversicherungskosten noch ein Mindestlohn bezahlt werden müssen. Welche Auswirkungen diese Form des Produktvertriebs auf Unternehmen mit angestellten Vertriebs- und Einzelhandelsbeschäftigten hat, ist bisher unerforscht. Angesichts der wachsenden Größenordnung des DV stellt sich jedoch sehr wohl die Frage, ob sich nicht ein die Arbeitsstandards drückender Effekt entwickeln könnte, wie er sich etwa auch beim Einsatz von Leiharbeitskräften im Hinblick auf Arbeits- und Gesundheitsschutzstandards zeigt (Becker 2015). Umso relevanter erscheint uns die Frage, welche Möglichkeiten es gibt, diese Situation zu ändern (hierzu Abschnitt 7). Dafür analysieren wir im nächsten Abschnitt zunächst die Gründe für die fehlende kollektive Interessenvertretung im DV.

\section{Fehlende kollektive Interessen- vertretung im Direktvertrieb}

Unsere bisherige Analyse hat die wachsende wirtschaftliche Bedeutung des Direktvertriebes wie auch die dort vorherrschenden ambivalenten bis hin zu prekären Arbeitsbedingungen gezeigt (vgl. auch Koehn 2001). Doch anders als bei der parallel anwachsenden (Problem-)Gruppe der digitalen Crowdworker lassen sich momentan keine Initiativen einer kollektiven Vernetzung oder kollektiven Interessenvertretung erkennen. Wir sehen vier Gründe, warum trotz der vielseitigen Probleme keine Anstrengungen für eine bessere Regulierung unternommen werden - weder vonseiten der Beschäftigten noch von bestehenden Institutionen.

Erstens fallen die Beschäftigten schlicht zwischen die Stühle der gesellschaftlichen und arbeitsmarktpolitischen Institutionen: Sie sind formal keine Arbeitnehmer im Sinne des Betriebsverfassungsgesetzes, d. h. sie haben auch keinerlei Interessenvertretung.

Zweitens unterstützt die oben beschriebene Ausrichtung der Unternehmen auf Beschäftigte, die oft anderweitig sozial abgesichert sind, den Eindruck, dass kein arbeitsmarktpolitischer Handlungsbedarf besteht. Die in vielerlei Hinsicht prekären Arbeitsbedingungen führen nicht zwangsweise zu prekären Lebenslagen. Zudem sind die Einflüsse und Rückwirkungen der Arbeitsbedingungen im
DV auf Einzelhandels- und Vertriebsunternehmen mit angestellten Beschäftigten unbekannt.

Drittens handelt es sich bei den Beschäftigten im DV um eine Gruppe, deren Mitglieder oft ein zu geringes soziales und ökonomisches Kapital besitzen, um eine eigene Interessenvertretung zu organisieren. Neben materiell abgesicherten Menschen (z. B. Frauen, deren Mann einem abgesicherten Arbeitsverhältnis nachgeht), scheint die Tätigkeit im DV vor allem für diejenigen attraktiv, die auf dem ersten Arbeitsmarkt Schwierigkeiten haben, sei es wegen fehlender Berufsausbildung, langer Krankheit, fehlender Sprachkenntnisse oder langer Arbeitslosigkeit. Anders als bei Selbstständigen mit hohem Ausbildungsniveau (z. B. Ärzte, Wirtschaftsprüfer oder Unternehmensberater) erfordert die Tätigkeit im DV keinerlei Vorkenntnisse oder Qualifikationen. Mitglieder von besonders schutzbedürftigen Gruppen können ihre Interessen jedoch nicht selbst erfolgreich gegenüber (internationalen) Großkonzernen vertreten. Damit perpetuiert sich der prekäre Status. Die Unsicherheit der Tätigkeit, das geringe Verdienstniveau und die unzureichenden Rechtskenntnisse haben zur Folge, dass die Vertrieblerinnen vielfach nicht über die nötigen Machtressourcen verfügen, die für die Gründung einer Interessenvertretung i. d. R. nötig sind.

Viertens tragen die im DV vermittelten unternehmenskulturellen Anrufungen dazu bei, dass das prekäre Potenzial der Arbeits- und Beschäftigungsbedingungen zunächst nicht offenkundig wird: Die Tätigkeit im DV wird zwar als Beschäftigung, (zweite) Karrierechance und gelegentlich selbst als Möglichkeit, großen Reichtum zu erlangen, präsentiert (Biggart 1989; Groß 2008; Groß/Haunschild 2013). Gleichzeitig knüpft die Tätigkeit in dreierlei Hinsicht an den privaten Bereich an, was den Beschäftigungscharakter verschleiert (Williams/Bermiller 2011): die Verkaufstätigkeit findet im privaten Raum statt, private Netzwerke werden für berufliche Zwecke genutzt und bei den meisten Produkten handelt es sich um Waren für den häuslichen Bereich, z. B. Haushaltswaren, Kosmetik, Nahrungsergänzungsmittel, Wellness und Wohnaccessoires. Hinzu kommt, dass zur Unternehmenskultur im DV die Botschaft gehört, dass jedes Mitglied selbst verantwortlich ist für seinen Erfolg und dementsprechend auch selbst die Verantwortung dafür zu tragen hat, wenn der Erfolg ausbleibt. Der von den Beschäftigten weitgehend internalisierte Anreiz, dass es ,jeder schaffen kann“, falls genug Anstrengungen unternommen werden, führt dazu, dass frühere Mitglieder sich ihren Misserfolg weitgehend selbst zuschreiben. Die strukturellen Ursachen (z. B. benötigter Zeitaufwand, geringfügige Bezahlung/ Provisionsstruktur, Marktsättigung, Konkurrenz der DV untereinander etc.) geraten damit aus dem Sichtfeld (Groß 2008; Groß/Haunschild 2013) und verringern das Risiko für die Unternehmen, dass Beschäftigte arbeitspolitische Forderungen aus den problematischen Arbeitsbedingungen ableiten. 


\section{Was sollte sich ändern?}

Die Konsequenz der beschriebenen Probleme besteht vor allem darin, dass der DV einer Regulierung und Kontrolle bedarf. Wir plädieren dafür, dass den Beschäftigten „,von außen“ geholfen wird, da die Wahrscheinlichkeit, dass Vertrieblerinnen sich selbst mobilisieren, eher gering (und de facto bisher nicht erfolgt) ist. Zwar könnte eine weitere Erosion des „male breadwinner model“ dazu führen, dass die Tätigkeit auch für die momentan größte Zielgruppe an Beschäftigten (Hausfrauen und Mütter) zunehmend unattraktiv (weil nicht existenzsichernd) wird und die Versprechen der Konzerne kritisch hinterfragt werden. Angesichts der Wachstumsraten des DV scheint dies momentan jedoch nicht der Fall zu sein. Daher sind staatliche Eingriffe, die auf Transparenz und Haltelinien zielen, gefragt. Gewerkschaften könnten Mobilisierungsbeispiele aus anderen Branchen (im Sinne von „best practice“) auch für den DV entwickeln und Unterstützung bei einer kollektiven Interessenartikulation bieten. Wir sehen zwei konkrete Maßnahmen zur Verbesserung der Arbeitsbedingungen im DV: mehr Transparenz und eine unabhängige Regulierungs-, Informations-, Beschwerde- und Kontrollstelle.

Vielen Arbeitsuchenden - und der breiteren Öffentlichkeit - fehlen Informationen zu den (auch langfristig wirkenden) Konditionen der Arbeit im DV: Weder ist bekannt, wie viele Unternehmen in der beschriebenen Art und Weise arbeiten, noch wie viele Mitglieder sie im Vertrieb haben, was diese verdienen, wie lange die Beschäftigten dabei sind bzw. wie schnell und aus welchen Gründen sie wieder ausscheiden. Wie andere Unternehmen könnte der DV verpflichtet werden, relevante Kennzahlen zu veröffentlichen. Unternehmen wie Herbalife oder Vemma, die durch die Federal Trade Commission (USA) untersucht wurden, wird genau dies auferlegt: die Veröffentlichung von Zahlen und Fakten, die Arbeitsuchenden Entscheidungshilfen bieten.

Bisher gibt es keine unabhängige Regulierungs-, Informations-, Beschwerde- und Kontrollstelle, die Unternehmen zurechtweisen kann bzw. tatsächlich effektiv zurechtweist. Zwar gibt es gesetzliche Vorgaben zu Schneeballsystemen und illegales Verhalten wird durch die Staatsanwaltschaft geahndet, die meisten Probleme des DV liegen aber entweder im Graubereich zwischen legal und illegal oder sind durch die Gesetzgebung gar nicht erfasst, wie eine aktuelle Analyse der Lobbypolitik des DV auf europäischer Ebene zeigt (Tokaji-Nagy 2016). Die Regeln für gutes Verhalten, die sich der DV über seine (inter-)nationalen Vereinigungen selbst gegeben hat (Codes of Ethics), haben sich als zu ungenau und wenig wirksam erwiesen, da sie nicht von einer unabhängigen Instanz überprüft werden.

Die genaue Ausgestaltung einer solchen Instanz für den DV kann viele Formen annehmen. Denkbar wäre z. B. eine unabhängige Kontrollstelle beim Ministerium für Arbeit und Soziales: So wie dort Regeln zu Leiharbeit und Werkverträgen aufgestellt werden, so müssten auch DV-spezifische Regeln und Vorgaben entwickelt werden, deren Einhaltung auch kontrolliert wird. Eine für den DV spezifische Instanz könnte nicht nur den Beschäftigten im DV „von außen“ helfen, sondern auch die Selbstwahrneh-

17 Für eine Erläuterung der Kriterien für „arbeitnehmerähnliche Personen“ siehe Borzaga (2012).

18 Auch die ver.di-Initiative "Dienstleistungspolitik 4.0" nimmt dieses Beschäftigungssegment nicht in den Blick. mung als Gruppe von Beschäftigten (statt „Selbstständigen“) stärken und somit zur Selbstmobilisierung der Beschäftigten beitragen. In diesem Zusammenhang könnte auch überprüft werden, ob bei den Beschäftigungsverhältnissen nicht eine Form der Scheinselbstständigkeit vorliegt. Ebenso stellt sich für den DV, der aus unserer Sicht zur Gruppe der „ertragsschwache[n] Arbeitsbeziehungen“ (Walter 2010) gehört, die Frage einer Neuregulierung des Arbeitsrechts. So wäre beispielsweise denkbar, Mitglieder des DV genauso wie unter bestimmten Bedingungen Handelsvertreterinnen und Handelsvertreter ( $\$ 92 \mathrm{a}$ HGB) zur Gruppe der „arbeitnehmerähnlichen Personen“ zu zählen und ihnen damit den Zugang zu Mindestarbeitsbedingungen zu gewähren. ${ }^{17}$

Die Rolle der Forschung ist in diesem Zusammenhang deutlich: Der DV wird bisher stiefmütterlich behandelt. Zwar gibt es für Deutschland einzelne Unternehmensstudien. Die Anzahl derjenigen, die dort tatsächlich einer Beschäftigung nachgehen, ihre Motive, die Bedingungen ihrer Tätigkeit, ihr Verdienst, ihre Aufstiegsmöglichkeiten, der Effekt ihrer Tätigkeit auf ihre (nicht aufgebaute) Altersrente, Karrierechancen außerhalb des DV etc. - also die Beschäftigungsbedingungen und die (sozio-ökonomischen sowie die individuellen) Folgen der Beschäftigung sind dagegen weitgehend unerforscht. Auch zu der oben aufgeworfenen Frage, welche Folgen das Wachstum des DV auf Vertriebs- und Einzelhandelsunternehmen mit angestellten Beschäftigten hat, sind uns keine Initiativen bekannt, die die genannten Lücken zu schließen versuchen. ${ }^{18}$

Für eine - aus unserer Sicht gebotene - Verbesserung der Beschäftigtensituation, das zeigen etwa die Erfahrungen mit Leiharbeit, muss dem DV erst mehr Aufmerksamkeit geschenkt werden: in der Öffentlichkeit, der Forschung und der Politik. Ziel unseres Artikels ist es, hierzu beizutragen.

\section{LITERATUR}

Becker, K. (2015): Macht und Gesundheit. Der informelle Handel um die Vernutzung von Arbeitskraft, in: Berliner Journal für Soziologie 25 (1), S. 161-185 Becker, K. (2016): Freiheitsfeten oder Prekaritätspartys? Tupperware als Erwerbsform von Frauen, in: Arbeits- und Industriesoziologische Studien 9 (1), S. $102-117$

Biggart, N. W. (1989): Charismatic capitalism. Direct selling organizations in America, Chicago

Borzaga, M. (2012): Wirtschaftlich abhängige Selbständige in Italien und Deutschland: eine rechtsvergleichende Analyse, in: Busch, D./Feldhoff, K./ Nebe, K. (Hrsg.): Übergänge im Arbeitsleben und (Re) Inklusion in den Arbeitsmarkt, Baden-Baden, S. 99-112

Brodie, S./Stanworth, J./Wotruba,T. (2002): Comparisons of salespeople in multilevel vs. single level direct selling organizations, in: Journal of Personal Selling \& Sales Management 12 (2), S. 67-75

Castel, R. (2000): Die Metamorphosen der sozialen Frage. Eine Chronik der Lohnarbeit, Konstanz

DSN (2012): Direct Selling News. 100 Global. Our exclusive annual report, Plano US

European Union (2013): Social protection rights of economically dependent self-employed workers, DG Internal Policies of the Union Policy Department A - Economic and Scientific Policy, Brussels

Groß, C. (2008): Multi-Level-Marketing: Identität und Ideologie im NetworkMarketing, Wiesbaden

Groß, C./Haunschild, A. (2013): Liberté toujours? Gerechtigkeit und Gemeinschaft in neoliberalen Arbeitsregimen am Beispiel der Amway $\mathrm{GmbH}$, in: Zeitschrift für Personalforschung 27 (2), S. 81-102

Koehn, D. (2001): Ethical issues concerned with multi-level marketing schemes, in: Journal of Business Ethics 29 (1/2), S. 153-160

Lan, P.-C. (2002): Networking capitalism: network construction and control effects in direct selling, in: The Sociological Quarterly 4 (2), S. 165-184 
Langreiter, N. (2006): Party Verkauf. Über modernes Hausieren am Beispiel von Tupperware, in: L'Homme Zeitschrift für Geschichtswissenschaft 17 (2), S. $119-133$

Manske, A./Merkel, J. (2009): Prekäre Freiheit - Die Arbeit von Kreativen, in: WSI-Mitteilungen 62 (6), S. 295-301, http://www.boeckler.de/wsi-mitteilungen 25255_25266.htm

Mayer-Ahuja, N. (2003): Wieder dienen lernen?: Vom westdeutschen "Normalarbeitsverhältnis" zu prekärer Beschäftigung seit 1973, Berlin

Peterson, R. A./Wotruba, T. R. (1996): What is direct selling?-Definition, perspectives, and research agenda, in: Journal of Personal Selling \& Sales Management 16 (4), S. 1-16

Schulze Buschoff, K. (2011): Gute Arbeit und „neue Selbständigkeit”, in: Bandelow, N. H./Hegelich, S. (Hrsg.): Pluralismus - Strategien - Entscheidungen, Wiesbaden, S. $406-421$

Tokaji-Nagy, O. J. (2016): A legal and empirical investigation into the direct selling industry's advocacy in the EU: Univeritaire Pers Maastricht,

https://cris.maastrichtuniversity.nl/portal/files/1419198/guid-cced183a-aa5845a0-b8d1-cdf5892d1aef-ASSET1.0

Walsh, J. (1999): How Ponzi Schemes, Pyramid Frauds work. And why they are more common than ever, in: Consumers' Research Magazine 82 (6),

S. $10-14$

Walter, T. (2010): Zum 68. Deutschen Juristentag: das Gutachten „Abschied vom Normalarbeitsverhältnis? - Welche arbeits-und sozialrechtlichen Regelungen empfehlen sich im Hinblick auf die Zunahme neuer Beschäftigungsformen und die wachsende Diskontinuität von Erwerbsbiographien?" von Prof. Dr.

Raimund Waltermann, in: Arbeit und Recht 58 (9), S. 364-367

WFDSA (World Federation of Direct Selling Associations) (2014): Global Direct

Selling - 2013 Retail Sales Vol. 2016

Williams, S. L./Bermiller, M. (2011): Women at work: Tupperware, passion

parties, and beyond, London

\section{AUTORINNEN}

CLAUDIA GROß, Dr., Assistant Professor, Institute for Management Research, Radboud University, Niederlande. Arbeitsschwerpunkte: Organisationsgestaltung, Unternehmensethik, Direktvertriebsunternehmen.

c.gross@fm.ru.nl

KARINA BECKER, Dr., Soziologin, wissenschaftliche Geschäftsführerin des DFG-Kollegs „Postwachstumsgesellschaften“, Friedrich-Schiller-Universität Jena. Arbeitsschwerpunkte: Wandel von Arbeit, Prekarität, Mitbestimmung.

karina.becker@uni-jena.de 